\title{
Translation, cross-cultural adaptation and validation of English start back screening tool into Bangla for patients with low back pain
}

\author{
Md. Israt Hasan and Syed Mozaffar Ahmed
}

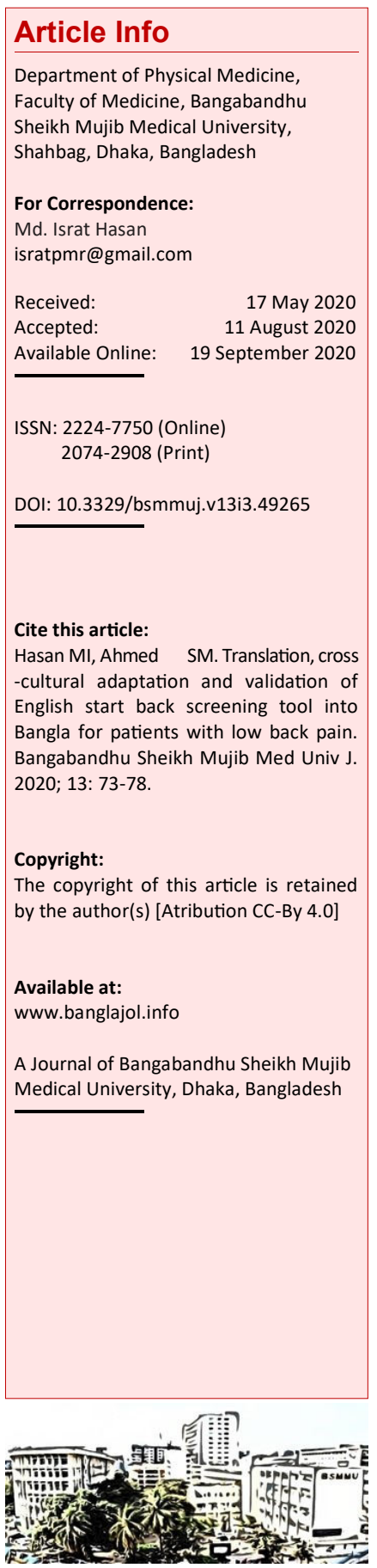

\section{Abstract}

This study was intended to translate and culturally adapt the STarT back screening tool to produce an equivalent Bangla version. Total 58 patients with low back pain completed the newly developed Bangla version of STarT back screening tool and Bangla version of Ronald Morris Disability Questionnaire seven days apart. Reliability was assessed by internal consistency (Chronbach's alpha for overall score 0.81 and for spychosocial subscale was 0.76 ) and test-retest reliability (intraclass correlation coefficient for overall score was 0.78 and for spychosocial subscale was 0.71 ). Reliability of Bangla version of STarT back screening tool was very good. Pearson's correlation coefficient was carried out on the Bangla version of STarT back screening tool and Bangla version of RMDQ to assess construct validity (overall score was 0.88 and spychosocial subscale score was 0.83 ) which indicate a strong correlation between them. This study shows that the Bangla version of STarT back screening tool is a reliable, valid and culturally adapted responsive screening tool for the patients with low back pain.

\section{Introduction}

Low back pain is a costly musculoskeletal problem and major public health issue in today's economically advanced world.1 Low back pain may interpret as aches and pains which affects the area between the lower border of the 12th rib and gluteal folds with or without radiation to the lower limb.2 Low back pain is among the top 10 diseases and injuries according to The 2010 Global Burden of Disease Study which is the highest number of disability-adjusted life year worldwide. $\underline{3}$ Standardized self-reported instruments are very user-friendly way to collect a large amount of data on activity limitation. 4 The STarT back screening tool is a validated and popular instrument in clinical practice and research. The original version of the STarT back screening tool is now available in the number of versions like English (original), $\underline{5}$ Arabic (Saudi Arab), $\underline{6}$ Chinese,,- Danish,, Dutch, $\underline{9}$ Finnish, 10 French,,$\underline{11}$ German, $\underline{12}$ Iranian, $\underline{13}$ Italian, $\underline{14}$ Brazillian, $\underline{15}$

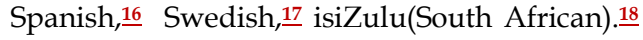
A substantial number of papers have been published on the psychometric properties, validity and reliability of the STarT back screening tool. Its' wide use in different studies carried out worldwide, have convinced the need to develop a version to be used in Bangladesh. The importance for translation, cross-cultural adaptation and validation of
STarT back screening tool from English to Bangla arises among the Bangla speaking people with low back pain due to growing international collaboration in clinical research and disability for proper rehabilitation program. The objective of this study was to develop a culturally adapted Bangla version of STarT back screening tool for use in Bangla speaking people. The developed Bangla version was applied in Bangladeshi patients with low back pain to study its acceptability, reliability and validity.

\section{Materials and Methods}

\section{The STarT back screening tool}

The STarT back screening tool is a validated simple, brief, and practical way to subgroup patients with nonspecific low back pain in primary care. The new STarT back screening tool identifies potentially modifiable prognostic indicators that may be appropriate targets for primary care interventions. $\frac{5}{5}$

The STarT back screening tool's appeal is obvious. It is short, easy to complete (most responses yes/no format), and quick to score. It can be easily implemented into busy clinical practice by health care practitioners. The care pathway for each subgroup is well-defined. The low risk subgroup is appropriate for self- 
management advice/education, reassurance, analgesics, and need re-consult only if necessary. The medium risk subgroup is appropriate for referral to physiotherapy.

The high risk subgroup should be referred to an appropriately skilled Rehabilitation physician to address psychological and physical obstacles to recovery. The keele STarT back screening tool is a 9item, self-reported questionnaire that includes treatment modifiable domains (spread of pain, disability, and psychological factors). It subgroups patients with non-specific low back pain into low, medium, and high risk of future disability with the purpose of matching each subgroup to a care pathway.

\section{The adaptation process}

The STarT back screening tool was adapted to the Bangladeshi population according to the guidelines of the Beaton et al forward-backward method. 19 Two bilingual translator independently translated the original English version of STarT back screening tool into Bangla. The independent translation matched absolutely. The two resulting Bangla draft versions were then synthesized into a single Bangla version by two translators. This version was then given to a third researcher, an independent native English speaker fluent in Bangla for back translation into English. The back translation matched the original version. To remove any comprehension barriers, the pre-final Bangla version was tested on

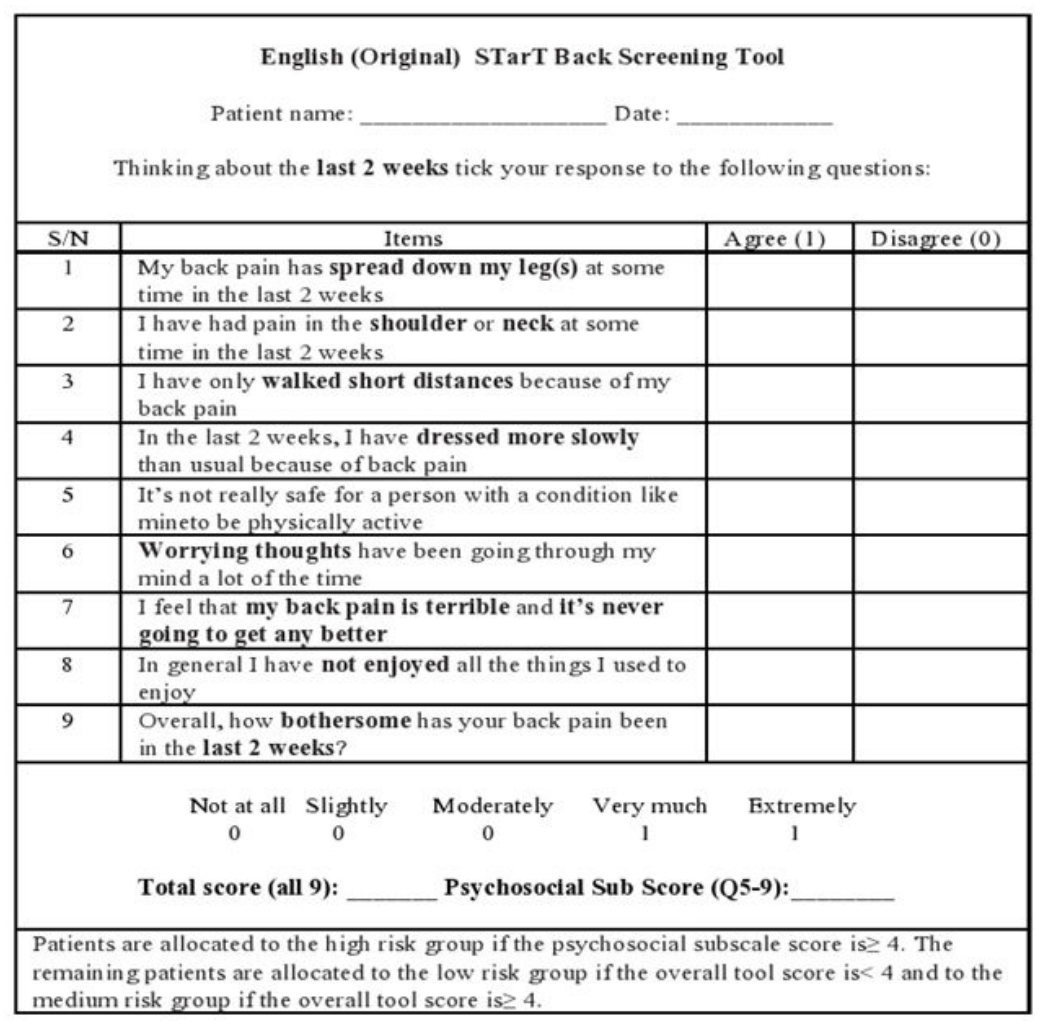

Figure 1. English (Original) SBST
20 volunteer patients to guarantee that participants would define each item as originally intended. The final Bangla version was then evaluated to verify its reliability and validity.

\section{Participants}

Inclusion criteria: Native Bangladeshi patients who are diagnosed with low back pain by a physician, aged from 18 to 80 years, and whose mother language were Bangla.

Exclusion criteria: Patients with tumor, ankylosing spondylitis, spinal fracture, history of surgery, and with comorbid serious systemic diseases were excluded. Patients who are unable to fill the questionnaire due to visual, cognitive, literacy related problems are also excluded.

\section{Psychometric Properties}

\section{Reliability}

Internal consistency: Bangla version of STarT back screening tool was tested in 58 patients to yield internal consistency by Cronbach's alpha. A Cronbach's alpha of 1.0 indicates a perfect correlation among all items, whether $\geq 0.7$ indicates satisfactory internal consistency.

Test-retest reliability: For test-retest reliability, the Bangla version of STarT back screening tool was served to consecutive subjects of 58 low back pain patients after being enrolled along with their informed consent where the participants were requested to fill questionnaire again after 7 days of the first enrollment without any medications adjustment in between. Intraclass correlation coefficient was used to assess the test-retest reliability. The intraclass correlation coefficient was expected to be found $\geq 0.70$; if so considered as acceptable for testretest reliability.

\section{Validity}

Construct validity: Construct validity was assessed by correlating the Bangla version of Roland Morris Disability Questionnaire (RMDQ)는 with Bangla version of STarT back screening tool and examined by Pearson correlation coefficient. RMDQ is a widely used outcome measure which showed excellent responsiveness in Bangladeshi population. Correlation coefficient were rated as follows: Strong correlation $>0.6$, moderate $\leq 0.6$ and $>0.3$ and poor $\leq 0.3$.

\section{Analysis}

Cronbach's alpha was used to evaluate internal consistency, on the other hand Intraclass correlation coefficient was used to assess the test-retest reliability. Construct validity was assessed by correlating the RMDQ with Bangla version of STarT back screening tool and examined by Pearson correlation coefficient. All statistical analyses were performed using SPSS version 24.0. A p value less 
than 0.05 was considered statistically significant.

\section{Results}

Among our study population just more than 20 were female and 38 were male. The mean age of which was $37.4 \pm 12.4$ years, for the most part of them were middle aged and older. Regarding literacy level, 6 were illiterate, 12, 14 and 19 went up to primary, secondary and higher secondary level respectively and 7 were graduate. Regarding profession most of them (21) were housewives, 7 were businessmen, 9 were garment worker, 5 were teacher, 6 were rikshaw puller, 4 were retired person, 4

\begin{tabular}{|c|c|c|c|}
\hline \multicolumn{4}{|c|}{$\begin{array}{l}\text { Bangla STarT Back Screening Tool (B-SBST) } \\
\text { রোগীর নাম: } \\
\text { বিগত দুই সপ্তাহের রথা চিন্তা করে, নিচের প্রশগুলোতেত টিৎ দিনঃ }\end{array}$} \\
\hline ক্রমিক নং & প্রশ্নমালা & একমত (১) & দ্বিমত (০) \\
\hline $\mathrm{s}$ & $\begin{array}{l}\text { আমার কোমড়ের ব্যথা গত দুই সপ্তাহের কোন না কোন সময়ে পায়য় } \\
\text { নেডেছে }\end{array}$ & & \\
\hline ₹ & $\begin{array}{l}\text { গত দুই সপ্তাহের কোন না কোন সময়ে আমার ঘাড়ে বা কার্ধে ব্যথা } \\
\text { ছিলো }\end{array}$ & & \\
\hline 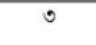 & 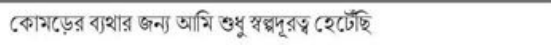 & & \\
\hline 8 & $\begin{array}{l}\text { গত দুই সপ্তারে কোমড়ের ব্যারার জন্য অন্য যেরোন সময়ের তুলনায় } \\
\text { অনেক ধীরে পোষাক পরেছি }\end{array}$ & & \\
\hline \& & আসার এই অবস্থায় শারীরিক ভাবে সচল থাকা নিরাপদ না & & \\
\hline ৬ & আসার মনে এ নিয়ে অনেক সময় দুশ্চিন্তা এসেছে & & \\
\hline 9 & $\begin{array}{l}\text { আগি মনে করি আমার কোমড়ের ব্যথা ভয়াবহ এবং এটা কখনো ভালো } \\
\text { হবার নয় }\end{array}$ & & \\
\hline $\mathrm{b}$ & $\begin{array}{l}\text { স্বাভাবিক ভাবে আমি যেসব কাজ উপভোগ করি তার কিছুই করতে } \\
\text { পারিনি }\end{array}$ & & \\
\hline ৯ & $\begin{array}{l}\text { লোটের উপর,গত দুই সপ্তাহে আপনার কোমড়ের ব্যথা কতোটা } \\
\text { অসুবিষাজনক ছিলো ? }\end{array}$ & & \\
\hline & 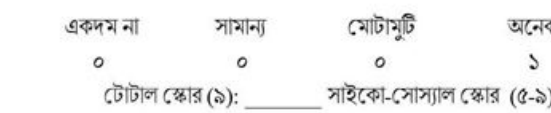 & $\begin{array}{l}\text { जीব्र } \\
د\end{array}$ & \\
\hline গাইকো-লো & 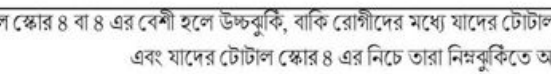 & $\begin{array}{l}\text { कार } 8 \text { वा } 8 \text { এর } 6 \\
\text { न }\end{array}$ & চারা মধামবুকি \\
\hline
\end{tabular}

Figure 2: Bangla version of STarT back screening tool were unemployed, and 2 were guard.

Translation process

We didn't come across any major linguistic or cultural barrier during forward and back translations. There was some minor inconsistencies. 6 of our patients were rikshaw puller and they were illiterate. For them some words had no equivalent in Bangla and were translated to the closest possible meaning, such as 'short distance' in questions 3, 'worrying' in question 6, 'terrible' in question 7 , 'enjoy' in question 8 and 'bothersome' in question 9. Certain phrases such as 'spread down' in question 1 and 'physically active' in question 5 posed difficulties for both forward translators.

But after finalize the Bangla version of STarT back screening tool almost all of our participants had no intricacy to recognize the translated items, and all of them responded to all components of the Bangla version of STarT back screening tool .

\section{Psychometrics \\ Reliability}

Internal consistency (Cronbach's alpha): Internal consistency with Cronbach's alpha for Bangla version of STarT back screening tool instrument is 0.81 for overall score and 0.76 for psychosocial subbscale. Alpha should be $>0.7$, which is standard for all scores. $\underline{\underline{21}}$

\section{Test-retest reliability}

Intra-class correlation co-efficient of Bangla version of STarT back screening tool: Calculated overall tool score is 0.78 and Psychosocial subscale score is 0.71 which signified substantial reliability. $\underline{.2}$

\section{Validity}

Construct validity: The construct validity of Bangla version of STarT back screening tool is presented in Table I, which demonstrated that the overall tool score is 0.88 and psychosocial score is 0.83 against reference standard RMDQ. All of the items were statistically significant $(\mathrm{p}<0.001)$.

\section{Table I}

Reliability and validity of Bangla version of STarT back screening tool

\begin{tabular}{|c|c|c|c|c|c|}
\hline \multicolumn{4}{|c|}{ Reliability } & \multirow{2}{*}{\multicolumn{2}{|c|}{$\begin{array}{c}\text { Validity } \\
\text { Construct validity } \\
\text { expressed by Pearson's correla- } \\
\text { tion coefficient }\end{array}$}} \\
\hline $\begin{array}{r}\mathrm{Ir} \\
\text { express }\end{array}$ & $\begin{array}{l}\text { ach's alp } \\
\text { ach's }\end{array}$ & $\begin{array}{l}\text { Test-retest re } \\
\text { intra-class cc }\end{array}$ & $\begin{array}{l}\text { oressed by } \\
\text { o-efficient }\end{array}$ & & \\
\hline Overall & Psychosocial & Overall & Psychosocial & Overall & Psychosocial \\
\hline 0.81 & 0.76 & 0.78 & 0.71 & 0.88 & 0.83 \\
\hline
\end{tabular}




\section{Discussion}

The STarT back screening tool is one of the critically demanding and internationally renowned tools for screening low back pain patients and is called for its reliability, validity, development in different cultures, and responsiveness to clinical development.23 The Bangla version of STarT back screening tool takes approximately $10 \mathrm{~min}$ to be completed, so it doesn't foist any extra strain on patients and researchers.

Reliability and validity is the core point of quality assessment. A reliable instrument yields the Xerox result on diverse occasions. Test-retest and internal consistency are two main established facets of reliability as a measurement tool of a patient's evaluation. In our study Overall score (Cronbach's alpha 0.81) and psychosocial subscale (Cronbach alpha 0.76) were very good which quite fairly indicates that the internal consistency measures a single concept of the questions of a scale supported by the results from the English (Original), Portuguese, Iranian, Brazilian, and French version of the STarT back screening tool (Table II).

Same result in repeated application attested the true reflection of Test-retest reliability. $\underline{24}$ Bangla version of STarT back screening tool brings in a excellent result in test-retest repeatability with Intraclass Corelation coefficients for overall score is 0.78 which was as good as English (original), isiZulu (South African), Portuguese, Finnish, Norwegian and Chinese versions of STarT back screening tool and for psychosocial score is 0.71 which was comparable to English (original), Portuguese and Norwegian version of STarT back screening tool .
An instrument is refers to be a valid one, when it measures what it is intended to measure. Bangla version of STarT back screening tool were correlated with Bangla version of RMDQ for the measurement of construct validity which was very good and statistically significant. In Bangla version of STarT back screening tool, overall score 0.88 shown strong correlation as like as English (original), Iranian, Swedish and Chinese version of STarT back screening tool in contrast to German version which shown moderate correlation. On the other hand, psychosocial score is 0.83 which shown strong correlation such as English (original) and Swedish version of STarT back screening tool compared to German version which shown moderate correlation.

\section{Conclusion}

The Bangla version of STarT back screening tool is a well translated and culturally adapted Bangla version of STarT Back Screening Tool as per internationally recommended guidelines. Findings suggest that reliability was very good and strong correlation with other psychometric instruments reflected relevant construct validity. We think that the Bangla version of STarT back screening tool will be an applicable and useful screening tool for clinical research in patients with low back pain.

\section{Funding Support}

Bangabandhu Sheikh Mujib Medical University research grant

Table II

Comparing reliability and validity of Bangla version of STarT back screening tool with different studies

\begin{tabular}{|c|c|c|c|c|c|c|c|}
\hline \multirow[t]{2}{*}{ Versions } & \multicolumn{2}{|c|}{ Chronbach } & \multicolumn{2}{|c|}{ Intraclass correlation coeffi- } & \multicolumn{2}{|c|}{ Validity } & \multirow{2}{*}{$\begin{array}{l}\text { Validity com- } \\
\text { paring tool }\end{array}$} \\
\hline & Overall & Psychosocial & Overall & Psychosocial & Overall & Psychosocial & \\
\hline Bangla & 0.81 & 0.76 & 0.78 & 0.71 & 0.88 & 0.83 & RMDQ \\
\hline English & 0.79 & 0.74 & 0.73 & 0.69 & 0.92 & 0,90 & RMDQ \\
\hline isiZulu & 0.68 & 0.68 & 0.73 & - & - & - & - \\
\hline Swedish & - & - & - & - & 0.61 & 0.60 & OMPSQ \\
\hline Portuguese & 0.93 & 0.91 & 0.74 & 0.76 & - & - & - \\
\hline Iranian & 0.82 & 0.79 & - & - & 0.81 & - & ODI \\
\hline Brazilian & 0.74 & 0.72 & - & - & - & - & - \\
\hline French & - & 0.74 & - & - & - & - & - \\
\hline Finnish & - & 0.52 & 0.78 & 0.68 & 0.45 & - & OMPSQ \\
\hline Norwegian & 0.51 & 0.58 & 0.89 & 0.82 & - & - & - \\
\hline German & - & - & - & - & 0.55 & 0.46 & RMDQ \\
\hline Chinese & - & - & 0.93 & - & 0.89 & - & RMDQ \\
\hline
\end{tabular}




\section{Ethical Issue}

This study was approved by the institutional review board of Bangabandhu Sheikh Mujib Medical University, (BSMMU/2019/8433) Dhaka, Bangladesh and all participants signed an informed consent to participate in the study.

\section{Conflict of Interest}

The authors declare no conflict of interest.

\section{Acknowledgement}

We are thankful to Dr Jonathan Hill for the permission to use the original version STarT Back Screening Tool for the translation, Cross-cultural adaptation and validation.

\section{References}

1. Hestbaek L, Leboeuf-Yde C, Manniche C. Low back pain: What is the long-term course? A review of studies of general patient populations. Eur Spine J. 2003; 12: 149-65.

2. Shakoor MA, Islam MT, Salek AKM, Moyeenuzzaman M. Effects of rehabilitation on the patients with chronic low back pain. Bangabandhu Sheikh Mujib Med Univ J. 2019; 12: 6-9.

3. Kaplan W, Wirtz VJ, Mantel-Teeuwisse. A priority medicines for Europe and the World 2013 Update.

4. Davidson M, Keating JL. A Comparison of five low back disability questionnaires: Reliability responsiveness. Phys Ther. 2002; 82: 8-24.

5. Hill JC, Dunn KM, Lewis M, Mullis R, Main CJ, Foster NE, Hay EM. A primary care back pain screening tool: Identifying patient subgroups for initial treatment. Arthritis Care Res. 2008; 59: 63241.

6. Elsabbagh L, Al-Atwi T, Aldossary D, Alshami AM, Hill JC, Abu-Ras W, Huijer HA, Brooks D. Cross-cultural adaptation and validation of the STarT back tool for Arabic speaking adults with low back pain in Saudi Arabia. J Orthop Sci. 2019; 24: 200-06.

7. Luan S, Min Y, Li G, Lin C, Li X, Wu S, Ma C, Hill JC. Cross-cultural adaptation, reliability, and validity of the Chinese version of the STarT back screening tool in patients with low back pain. Spine 2014; 39: E974-79.

8. Morsø L, Albert H, Kent P, Manniche C, Hill J. Translation and discriminative validation of the STarT back screening tool into Danish. Eur Spine J. 2011; 20: 2166-73.

9. Bier JD, Sandee-Geurts JJ, Ostelo RW, Koes BW,
Verhagen AP. Can primary care for back and/or neck pain in the Netherlands benefit from stratification for risk groups according to the STarT back tool classification? Arch Phys Med Rehab. 2018; 99: 65-71.

10. Piironen S, Paananen M, Haapea M, Hupli M, Zitting P, Ryynänen K, Takala EP, Korniloff K, Hill JC, Häkkinen A, Karppinen J. Transcultural adaption and psychometric properties of the STarT back screening tool among Finnish low back pain patients. Eur Spine J. 2016; 25: 287-95.

11. Bruyère $O$, Demoulin $M$, Brereton $C$, Humblet $F$, Flynn D, Hill JC, Maquet D, Van Beveren J, Reginster JY, Crielaard JM, Demoulin C. Translation validation of a new back pain screening questionnaire (the STarT back screening tool) in French. Arch Public Health. 2012; 70: 12.

12. Aebischer B, Hill JC, Hilfiker R, Karstens S. German translation and cross-cultural adaptation of the STarT back screening tool. PLoS One. 2015; 10: e0132068.

13. Azimi P, Shahzadi S, Azhari S, Montazeri A. A validation study of the Iranian version of STarT back screening tool (SBST) in lumbar central canal stenosis patients. J Orthop Sci 2014; 19: 213-17.

14. Maggiani A, Abenavoli A. Italian translation and cross-cultural adaptation of a back-pain screening questionnaire (STarT back screening tool). Annali di Igiene. 2019; 31: 69-75.

15. Pilz B, Vasconcelos RA, Marcondes FB, Lodovichi SS, Mello W, Grossi DB. The Brazilian version of STarT back screening tool: Translation, crosscultural adaptation and reliability. Braz J Phys Ther. 2014; 18: 453-61.

16. Gusi N, del Pozo-Cruz B, Olivares PR, HernándezMocholi M, Hill JC. The Spanish version of the "STarT back screening tool" (SBST) in different subgroups. Atencion Primaria. 2011; 43: 356-61.

17. Betten C, Sandell C, Hill JC, Gutke A. Crosscultural adaptation and validation of the Swedish STarT back screening tool. Eur J Physiother. 2015; 17: 29-36.

18. Schmidt PA, Naidoo V. Cross-cultural adaptation and validation of the STarT back screening tool in isiZulu. South African J Physiother. 2020; 76.

19. Beaton D, Bombardier C, Guillemin F, Ferraz MB. Recommendations for the cross-cultural adaptation of health status measures. Am Acad Orthop Surg. 2002; 12: 1-9.

20. Islam SM, Emran M, Baral AB, Happy BD, Islam $F$, Saha RK, Khan MM. Roland Morris disability questionnaire in Bengali for evaluation of patients with low back pain. KYAMCJ. 2020; 11: 21-25.

21. Dodds TA, Martin DP, Stolov WC, Deyo RA. A validation of the functional independence measurement and its performance among rehabilitation inpatients. Arch Phys Med Rehab. 1993; 74: 531-36. 
22. Cohen J. Statistical power analysis. Curr Dir Psychol Sci. 1992; 1: 98-101.

23. Azimi P, Shahzadi S, Azhari S, Montazeri A. A 24. Nunnally JC. Psychometric theory. 3rd ed. Tata validation study of the Iranian version of STarT back screening tool (SBST) in lumbar central canal stenosis patients. J Orthop Sci. 2014; 19: 213-17. 